\title{
Maschke, Lisa; Mießner, Michael; Naumann, Matthias (2021): Kritische Landforschung. Konzeptionelle Zugänge, empirische Problemlagen und politische Perspektiven
}

\author{
Jens Reda (1) \\ Eingegangen: 11. Februar 2021 - Angenommen: 19. Februar 2021 - Online veröffentlicht: 21. April 2021
}

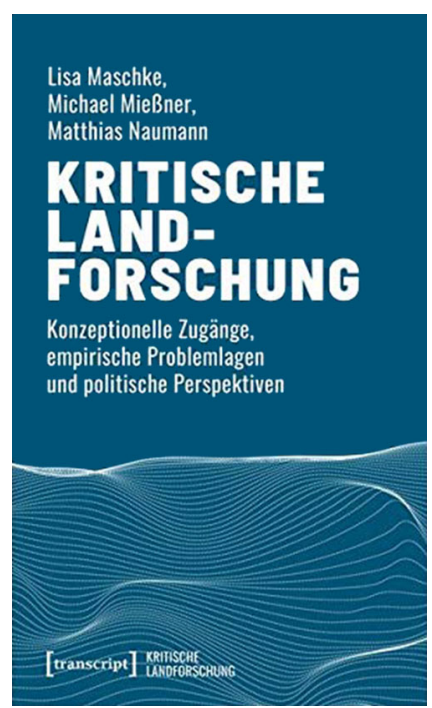

Wer von Städten spricht, kann vom Land nicht schweigen - so lässt sich der Ausgangsbefund der von Lisa Maschke, Michael Mießner und Matthias Naumann verfassten Studie beschreiben. Sie entwirft ein gesellschaftskritisches Forschungsprogramm, welches dem städtischen Fokus der gegenwärtigen kritischen Raumforschung im deutschspra-

$\square$ Jens Reda, M. Sc., Geographisches Institut, Christian-Albrechts-Universität zu Kiel, Ludewig-Meyn-Straße 14, 24118 Kiel reda@geographie.uni-kiel.de

(c) (1) (c) 2021 Reda; licensee oekom verlag. This Open Access article is published under a Creative Commons Attribution 4.0 International License. chigen Kontext eine rurale Perspektive zur Seite stellt. Doch warum in einem Zeitalter global zu beobachtender Verstädterungsprozesse überhaupt noch einen Blick auf das Land werfen? Medial wie politisch werden urbane Zentren als Triebfedern der politischen, ökonomischen und sozialen Entwicklungen verhandelt und damit verbundene Kämpfe um Wohnraum, öffentliche Infrastrukturen oder politische Partizipation als primär städtische Phänomene gerahmt. Wird doch einmal über das Land gesprochen, dann verweben sich die Narrative nicht selten zu einem Spannungsfeld aus idyllisierten Vorstellungen über eine entschleunigte und naturnahe Lebensweise und problematisierenden Befunden über periphere ,Resträume‘.

Die vorgelegte Monographie zur kritischen Landforschung, die zugleich den Auftakt einer gleichnamigen Schriftenreihe bei Transcript bildet, interveniert in dieses Spannungsfeld und eröffnet eine erfrischende Sichtweise auf ländliche Räume jenseits der Dichotomie aus Idylle und Niedergang. Denn als Produkte gesellschaftlicher Praxen und Strukturen stellen ländliche Räume komplexe Lebensund Wirtschaftsräume, Kreativ- und Naturräume dar und sind dabei ,nicht minder als Städte von den Auswirkungen der Globalisierung, vom Klimawandel, von Veränderungen der Arbeitswelt, demographischen Prozessen und politischen Verschiebungen durch die Dominanz neoliberaler Programme betroffen“ (S. 13). Tiefgreifende Analysen und gesellschaftstheoretisch fundierte Diskussionen dieser Prozesse sind bislang jedoch kaum Gegenstand der deutschsprachigen Geographie ländlicher Räume. Die wissenschaftliche Debatte hierzulande ist vielmehr durch kulturtheoretisch ausgerichtete Arbeiten über die symbolischen Aushandlungen des Ländlichen sowie stärker steuerungsund planungsorientierte Ansätze der Regionalentwicklung geprägt. Es ist daher eine große Qualität des nur 150 Seiten 
starken Buches, der Vielschichtigkeit ländlicher Entwicklungsprozesse aus einer dezidiert gesellschaftskritischen Perspektive nachzugehen.

In ihrem Bestreben, eine kritische Landforschung anzuregen, greifen die Autorin und Autoren auf entsprechende Ansätze der englischsprachigen Forschung zurück, die bislang vergleichsweise selten in den deutschsprachigen Kontext überführt wurden. In Form einer Literaturschau, die sich auf maßgebliche Einführungs- und Sammelbände sowie debattenprägende Zeitschriften stützt, werden zentrale Themen und Konzepte der anglophonen Debatte, allen voran der Rural Geography, identifiziert und strukturiert sowie prägnant dargestellt. Dabei sind sich die Autorin und Autoren ihrer selektiven Vorgehensweise sowie der Fokussierung auf den Globalen Norden durchaus bewusst. Entsprechend soll die Studie erste Anstöße für Studierende, Wissenschaftler/-innen und Aktivist/-innen liefern, sich weiterführend mit kritischen Perspektiven auf ländliche Entwicklungsprozesse auseinander- und für eine emanzipatorische Politik ländlicher Räume einzusetzen.

Hiervon ausgehend gliedert sich das Buch dann auch in drei zentrale inhaltliche Abschnitte. Im ersten Teil werden mögliche Konzepte einer kritischen Landforschung vorgestellt, die dazu beitragen können, ,allgemeine Muster ländlicher Entwicklung und deren Hintergründe zu verstehen, um lokal spezifische Bedingungen einordnen zu können“ (S. 23). Hierunter fallen kritisch-materialistische Theorien zur räumlich ungleichen Entwicklung, mit denen sich die strukturelle Benachteiligung ländlicher Räume durch urbane Eliten und global zirkulierende Kapitalströme eingehender herausarbeiten ließe. Des Weiteren werden die politisch-ökologischen Konzepte der Nahrungsregime sowie der Umweltgerechtigkeit als mögliche Analyserahmen angeführt. Sie ermöglichen es, die macht- und kapitalbezogene Formation ressourcenbezogener Produktionsprozesse sowie die gesellschaftlich ungleiche Verteilung damit verbundener Umweltfolgeschäden in ländlichen Regionen genauer zu betrachten. Zuletzt werden hegemoniale Diskurse über das Land thematisiert. Hier wird den eingangs skizzierten Deutungsmustern der Idylle und Anti-Idylle das Konzept des dreifachen ländlichen Raumes von Keith Halfacree zur Seite gestellt, um die Beziehungsweisen gesellschaftlich zirkulierender Vorstellungen, alltäglicher Praktiken und politisch-ökonomischer Strukturen bei der sozialen Produktion ländlicher Räume aufzuzeigen. In der Darstellung dieser eher klassischen Ansätze der kritischen Raumforschung beschränken sich Autorin und Autoren auf wesentliche Kernaussagen, wodurch die Theorien einem breiten Publikum zugänglich werden. Ähnliche Verweise auf Theorieangebote wie die Assemblage-Theorie, die diese Arbeiten um eine stärker prozessorientierte Perspektive auf ländliche Entwicklungsfragen ergänzen könnten (Jones/
Heley/Woods 2019), hätten hier den inhaltlichen Rahmen erweitern können, die Diskussion aber auch komplexer gestaltet.

Aufbauend auf die Theorieangebote rücken im zweiten Abschnitt aktuelle Themen einer kritischen Landforschung in den Fokus. Auch hier folgen die Autorin und Autoren einer klaren Struktur und fächern die Inhalte entlang der Felder ländliche Ökonomien, sozialer Wandel, Mensch-Umwelt-Beziehungen und Machtverhältnisse in ländlichen Räumen auf. Die Themenbereiche sind selektiv und speisen sich aus verschiedenen regionalen Studien im Globalen Norden. Wenngleich den Leserinnen und Lesern damit manches Beispiel etwas fern vorkommen mag, so verdeutlichen diese doch die Relevanz und Aktualität, die kritische Forschungsperspektiven für Entwicklungen in den ländlichen Räumen Deutschlands haben. So werden neben Erläuterungen zum wirtschaftlichen Strukturwandel auch Debatten um Klimaanpassungsmaßnahmen sowie die Ausbeutung natürlicher Ressourcen aufgegriffen, die im Kontext einer global stattfindenden Kommodifizierung von Natur auch für ländliche Regionen in Deutschland von Bedeutung sind. Darüber hinaus finden Themen wie Armut, Rassismus und eine defizitäre Wohnraumversorgung einen prominenten Platz. Diese werden bisher kaum aus einer ländlichen Perspektive diskutiert, prägen jedoch auch hierzulande die Lebensrealitäten vieler Landbewohner/-innen. Begleitet werden all diese Themen von Fragen nach den gesellschaftlichen Machtverhältnissen, die sich nicht allein auf der Ebene der politischen Steuerung und Einflussnahme von ländlichen Entwicklungsprozessen zeigen. Es ist daher begrüßenswert, dass Autorin und Autoren neben ländlichen Entwicklungsparadigmen auch dem Erstarken populistischer Bewegungen in ländlichen Milieus ihre Aufmerksamkeit schenken.

Vor dem Hintergrund des kritischen Grundtenors liefern die Autorin und die Autoren im dritten Abschnitt Impulse für alternative ländliche Entwicklungsansätze. Angefangen mit Ansätzen der lokalen und regionalen Selbstorganisation, die teilweise bereits in ländlichen Regionen praktiziert werden (z. B. solidarische Landwirtschaften), werden hier auch radikaldemokratische Perspektiven aufgeworfen, die bislang eher auf Städte bezogen sind. Neben Gemeinschaftsgütern und einem ,Recht auf Dorf', das die grundlegende Mitbestimmung von Bewohnerinnen und Bewohnern über die Gestaltung ihres Lebensraums einfordert, ist hier die Reorganisation lokaler Regierungsstrukturen im Sinne munizipalistischer Zusammenschlüsse anzuführen. Die Vorstellung dieser Ansätze regt zum utopischen Denken an, bietet jedoch wenig Einblicke in deren Umsetzung. Dies mag der noch jungen Debatte um solche Konzepte für ländliche Kontexte sowie dem Charakter der Literaturschau geschuldet sein, doch wäre ein stärkerer Praxisbezug gerade in diesem Abschnitt wünschenswert gewesen. 
Die Relevanz emanzipatorischer Perspektiven auf ländliche Räume bleibt dennoch unbestritten. Gleichzeitig werfen die Autorin und Autoren im Fazit Fragen auf, denen sich eine kritische Landforschung zukünftig stellen muss. Dies betrifft zum einen die Frage, ob und in welcher Weise eine eigenständige kritische Theorie ländlicher Räume möglich und notwendig ist. Zum anderen ist danach zu schauen, welche Wirkung eine kritische Landforschung auf die Praxis der ländlichen Entwicklung entfalten kann.

Beide Fragen spiegeln den selbstformulierten Anspruch für das Buch wider, Impulse für eine kritische Landforschung sowie emanzipatorische Landpolitik zu geben. Die klare Struktur sowie verständliche Sprache, in welcher die Themen und Konzepte auf ihren inhaltlichen Kern hin umrissen werden, legen hierfür einen guten Grundstein. Gleichzeitig bietet der Charakter des Literaturberichts Anknüpfungspunkte für weiterführende Ideen. In diesem Zusammenhang wird es spannend sein zu sehen, inwiefern eine kritische Landforschung an aktuelle konzeptionelle Diskussionen der kritischen Raum- und Stadtforschung anschließen und hierbei eigene Akzente setzen kann. Der von Lisa Maschke, Michael Mießner und Matthias Naumann formulierte Auftakt dieser Debatte sowie der aufgenommene Dia$\log$ zwischen Kritischer und Angewandter Geographie (Ku-
ge/Naumann/Nuissl et al. 2020) stimmen in jedem Fall optimistisch, dass das Projekt einer kritischen Landforschung auf fruchtbaren Boden fällt.

\section{Vollständige bibliographische Angaben des rezensierten Werkes:}

Maschke, Lisa; Mießner, Michael; Naumann, Matthias (2021): Kritische Landforschung. Konzeptionelle Zugänge, empirische Problemlagen und politische Perspektiven. Bielefeld: Transcript Verlag. $=$ Kritische Landforschung 1. 3 Abbildungen, 150 Seiten

\section{Literatur}

Jones, L.; Heley, J.; Woods, M. (2019): Unravelling the Global Wool Assemblage: Researching Place and Production Networks in the Global Countryside. In: Sociologia Ruralis 59, 1, 137-158. https://doi.org/10.1111/soru.12220

Kuge, J.; Naumann, M.; Nuissl, H.; Schipper, S. (2020): Angewandte und Kritische Geographie. Gemeinsame Herausforderungen, gemeinsame Perspektiven? In: Standort - Zeitschrift für Angewandte Geographie 44, 4, 219-225. https://doi.org/10.1007/s00548-020-00662-x 